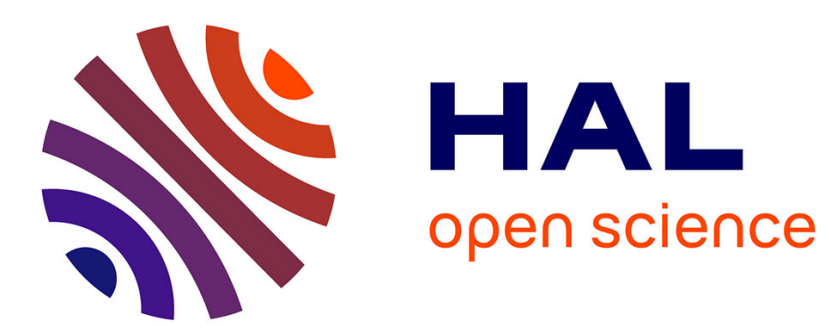

\title{
UN DISPOSITIF DE SENSIBILISATION PARENTALE DANS UN MUSEE SCIENTIFIQUE
}

Rodica Ailincai, Annick Weil-Barais

\section{To cite this version:}

Rodica Ailincai, Annick Weil-Barais. UN DISPOSITIF DE SENSIBILISATION PARENTALE DANS UN MUSEE SCIENTIFIQUE. Revue internationale de l'éducation familiale, 2006, 20, pp.87-108. hal00238549

\section{HAL Id: hal-00238549 \\ https://hal.science/hal-00238549}

Submitted on 4 Feb 2008

HAL is a multi-disciplinary open access archive for the deposit and dissemination of scientific research documents, whether they are published or not. The documents may come from teaching and research institutions in France or abroad, or from public or private research centers.
L'archive ouverte pluridisciplinaire HAL, est destinée au dépôt et à la diffusion de documents scientifiques de niveau recherche, publiés ou non, émanant des établissements d'enseignement et de recherche français ou étrangers, des laboratoires publics ou privés. 


\title{
UN DISPOSITIF DE SENSIBILISATION PARENTALE DANS UN MUSEE SCIENTIFIQUE
}

\author{
AILINCAI Rodica ${ }^{1}$, WEIL-BARAIS Annick ${ }^{2}$
}

\begin{abstract}
Résumé
A partir de la prise en considération de l'importance du rôle des parents accompagnant leurs enfants (35 ans) dans la découverte d'une exposition interactive à caractère scientifique et technique, la recherche propose : (1) la conception d'un dispositif de Sensibilisation Parentale (DSP) - et notamment d'un film, support de débats et de témoignages de la part des parents qui vise surtout une prise de conscience de la part du parent des liens entre sa conduite et celle de l'enfant ; (2) une évaluation du DSP. L'étude a été réalisée dans l'exposition des «3/5 ans » de la Cité des Enfants, à la Cité des Sciences et de l'Industrie à Paris en direction des parents intéressés par une «visite pédagogique» de l'exposition. Au total, 7 dyades parent-enfant ont été observées et leurs échanges transcrits et analysés en référence à différents modèles (actes tutoriels, pragmatique conversationnelle et actes de distanciation parentale). L'hypothèse selon laquelle une action de sensibilisation préalable des parents à leur rôle de tuteur a un impact sur la manière dont ils accompagnent leur enfant se trouve confirmée. Les analyses montrent en effet une évolution marquée et très individualisée entre leur première visite et leur seconde visite au musée, après que les parents aient été impliqués dans le DSP. Ces données sont discutées dans le contexte des études sur l'éducation parentale.
\end{abstract}

Mots-clés : éducation parentale - musée scientifique - interactions parent/enfant- jeunes enfants

A parents' training scheme - Raising parents' awareness of their guiding role for their children in a museum of science and technology

\begin{abstract}
:
Taking into account the importance of the role of parents in accompanying their children (3-5 years old) through the discovery of an interactive scientific and technical exhibition, this research offers : (1) the elaboration of a Parents Training Scheme (PTS) - aimed mainly at changing parents' attitudes towards their children, including a film to stimulate discussion and experience sharing among parents ; (2) an evaluation of the PTS. The study was conducted with parents interested in a "pedagogical visit" of the "3-5 years old" exhibition at the Cité des enfants ("Children's corner") of the Cité des Sciences et de l'Industrie (Paris). A total of 7 parent-child dyads were observed and their spoken exchanges transcribed and analysed in reference to different models (tutorial acts, conversational pragmatics, and parents' distancing acts). The hypothesis suggesting that previous awareness of their role as tutor modifies the way parents accompany their children is verified. Analyses show a sharp and individualised evolution from their first visit to the museum to their second one after the PTS. These data are discussed in relation to previous studies on parent training.
\end{abstract}

Key-words: parent training - science museum - learning - parent/child interaction

\section{Les expositions scientifiques pour enfants : un lieu privilégié d'éducation}

Les expositions scientifiques destinées aux enfants proposent un environnement interactif dans lequel le plaisir est associé à la découverte active des sciences et des techniques ; rendre les enfants curieux du monde qui les environne, en y associant le plaisir de la découverte et de la compréhension, est l'un des objectifs essentiels de ces expositions. Ces espaces sont

\footnotetext{
${ }^{1}$ UNIVERSITE RENÉ DESCARTES - PARIS 5, Education et Apprentissages, 45 rue des Saint Pères, 75006 PARIS Tel.: +33 142863370 - Fax:+33142863361 - r.ailincai@yahoo.fr

2 UNIVERSITE D'ANGERS, 11 boulevard Lavoisier, 49045 ANGERS Cedex 01 Tel.: 33 (0)2.41.22.64.21 - Fax : 33 (0)2.41.22.64.19 annick.weilbar@wanadoo.fr
} 
généralement conçus en référence à une approche socio-constructiviste du développement des connaissances.

Ainsi à la Cité des Enfants, à la Cité des Sciences et de l'Industrie (Paris), où le dispositif d'éducation parentale présenté dans cet article a été expérimenté, les adultes sont invités à exercer le rôle de médiateur. Les éléments d'exposition ont été conçus dans cette optique, et le fonctionnement organisé de façon à ce que les enfants soient toujours accompagnés par des adultes. Les études ultérieures concernant les interactions parent-enfant dans l'exposition destinée aux plus jeunes -âgés de 3 à 5 ans- ont montré que les modes d'intervention des parents sont assimilables à du tutorat (Ailincai et Weil-Barais, 2003, Ailincai et al., 2005). Bruner (1983) souligne que l'étayage de l'enfant par l'adulte dans la réalisation d'une tâche a des limites d'efficacité qui sont déterminées par les capacités actuelles de l'enfant: si l'activité proposée par l'adulte est trop éloignée du niveau réel de l'enfant, la progression ne pourra pas se faire. Ainsi les acquisitions dépendent à la fois du niveau déjà atteint par l'enfant et de l'aide instrumentale juste nécessaire.

La visite d'une exposition à caractère scientifique et technique étant souvent perçue comme ayant des objectifs éducatifs, le comportement de tutelle dépend souvent de la conception des parents sur la pédagogie (Guichard, 1998). Le modèle imaginé par les concepteurs de l'exposition lors de l'élaboration des dispositifs muséologiques de la Cité des enfants était de favoriser une « visite pédagogique non directive » : l'enfant questionne, agit, l'adulte explique à la demande... (Guichard, 1993). Plusieurs études concernant les interventions des parents dans l'exposition ont bien mis en évidence une multitude de modes d'accompagnements, plus ou moins favorables à la découverte de l'exposition; souvent les parents s'avèrent plus préoccupés des actions des enfants et de leur réussite que de la compréhension par ces derniers des mécanismes explorés (Chaumier et al., 1995, Piani \& Weil-Barais, 1993 ; Cauzinille-Marmèche \& Samuels-Pitzini, 1996 ; Ailincai \& Weil-Barais, 2003). Même si les conduites des parents ne sont pas toujours adaptées à la découverte des sciences et des techniques, qui suppose une approche réfléchie et contrôlée des dispositifs, ceux-ci semblent conscients de leur rôle d'éducateurs auprès de leurs enfants et ils sont demandeurs d'informations pour intervenir de manière favorable auprès des enfants (Ailincai, 2005). Ceci laisse à penser que les espaces muséologiques destinés aux enfants pourraient être des lieux privilégiés de formation des parents, l'environnement et le contexte muséal permettant d'échapper aux cadres normatifs assez fréquents en éducation parentale. Selon Pourtois (1984, 2004), l'éducation parentale est une activité volontaire d'apprentissage de la part des 
parents qui souhaitent améliorer les relations avec leur enfant, pour favoriser l'émergence de comportements jugés positifs et diminuer celle de comportements jugés négatifs. En effet, les parents vont au musée à leur convenance et ils sont seuls juges de leurs besoins et des bénéfices qu'ils tirent des activités auxquelles ils participent. Terrisse (1997), considère que ces formations ont pour but d'aider les parents à actualiser leurs potentialités éducatives, en développant leur sentiment de compétence et en utilisant le mieux possible les ressources que leur offre leur environnement. Les thèmes proposés par les expositions scientifiques, faisant souvent appel à des connaissances spécifiques et des compétences pédagogiques, justifient d'autant plus une telle formation en direction des parents.

Le présupposé qui est à la base du dispositif de sensibilisation des parents présenté dans cet article est que, dans nos sociétés modernes, du fait notamment des changements rapides et des ruptures intergénérationnelles, tous les parents ont besoin, à un moment ou à un autre, d'être accompagnés pour pouvoir assurer leur rôle d'éducateur auprès des enfants (Pourtois, Desmet et Nimal, 2000). Ainsi, les musées qui sont des lieux d'éducation par excellence et dont le prestige attire le public, pourraient offrir des dispositifs de formation des parents en accès libre.

Le dispositif que nous présentons dans ce chapitre a ainsi été conçu à titre expérimental afin de pouvoir en évaluer la pertinence. Nous présenterons tout d'abord ses caractéristiques ainsi que les présupposés qui le fondent puis nous exposerons les éléments utilisés à des fins d'évaluation. Nous discuterons enfin de la spécificité et de l'intérêt du dispositif proposé, ainsi que de ses limites dans une perspective d'extension.

\section{Description du dispositif}

Le dispositif a été conçu pour fonctionner pendant une durée limitée (une heure environ) dans le cadre du musée.

\subsection{Caractéristiques du dispositif}

Le dispositif vise à sensibiliser les parents qui le souhaitent à leur rôle éducatif, terme préféré à ceux d'éducation ou de formation car le dispositif vise surtout une prise de conscience du parent en l'incitant à être attentif aux liens entre ses interventions et les conduites de l'enfant. Nous attendons de ces prises de consciences des modifications des conduites du parent dans le sens d'une meilleure adaptation aux besoins de l'enfant.

Dans la pratique, les parents, en nombre limité (sept personnes dans le cadre de l'expérimentation rapportée dans cet article), ont participé à une réunion-discussion 
consécutive après avoir visionné un film restituant deux séquences très différentes du point de vue des styles interactionnels des dyades parent-enfant mais identiques du point de vue du cadre (même élément muséologique exploré, mêmes acteurs). La conception de ce film a fait l'objet d'un soin particulier car nous souhaitions qu'il présente des situations représentatives de celles que connaissent les parents. Par ailleurs, nous souhaitions que ces situations puissent être appréhendées facilement du point de vue des conduites des acteurs (l'adulte et l'enfant). Ces exigences correspondent aux fonctions accordées au film dans le dispositif de sensibilisation : support d'analyse des conduites et de la prise de conscience des liens entre les conduites de l'adulte et celles de l'enfant. Nous avons présupposé que le fait de visionner collectivement des séquences filmées, dans le cadre d'un espace phare du musée que tous les visiteurs explorent, permettrait à des parents qui ne se connaissent pas de partager un contexte mutuel facilitant les échanges ultérieurs.

\subsection{Présentation du film}

Le film d'une durée de projection de sept minutes présente deux séquences au cours desquelles un enfant et un adulte interagissent (cf. Encadré 1). Elles ont été conçues à partir d'une étude préalable des pratiques effectives des parents (Ailincai \& Weil-Barais, 2003).

Premier scénario (en référence au style interactif directif, - cf. Encadré 2, conduite 1.2)

Le parent montre à l'enfant comment il faut faire et impose des actions sans les expliquer. C'est plutôt le parent qui manipule et qui semble satisfait.

L'enfant change souvent de place pour manipuler seul, sans se soucier de l'adulte; ensuite il abandonne. Le parent et l'enfant ont passé une minute sur le dispositif et c'est plutôt le parent qui a manipulé.

Deuxième scénario (en référence au style interactif suggestif - cf. Encadré 2, conduite 2.1)

Le parent incite l'enfant à rechercher des relations causales en formulant des demandes qui incitent à la réflexion. Le parent fait verbaliser l'enfant.

L'enfant oriente l'eau en vue de faire tourner un moulin particulier. L'objectif de la manipulation est atteint. Le parent et l'enfant ont passé quatre minutes sur le dispositif.

Encadré 1 : les scénarios porposés pour la formation des parents.

Les deux séquences du film sont interprétées par deux acteurs, un adulte et un enfant, afin de centrer l'attention des participants sur les actions de ces acteurs et non sur des aspects personnels. Nous proposons également une unité de lieu (le dispositif « Orienter l'eau » ${ }^{3}$ ), les différents dispositifs ne se prêtant pas tous aux mêmes démarches d'exploration. Les deux séquences présentées ont été sélectionnées à partir d'un ensemble de cinq séquences

\footnotetext{
${ }^{3}$ Ce dispositif, constitué d'un plateau rectangulaire positionné en plan incliné, présente un ensemble de canaux sur lesquels se trouvent des moulins colorés. Les enfants ont la possibilité d'actionner plusieurs manettes permettant le changement de direction du cours de l'eau ; ce changement entraîne le mouvement des moulins positionnés sur le trajet.
} 
initialement conçues, correspondant à l'ensemble des conduites préalablement repérées et ayant débouché sur une typologie des styles d'intervention (Ailincai \& Weil-Barais, 2003) (cf. Encadré 2).

\section{Style interactif directif :}

Conduite 1.1 Les parents qui décrivent à l'enfant le dispositif, sans lui expliquer le fonctionnement - ce type d'interaction correspond souvent à la catégorie des parents qui considèrent avoir un rôle de "guide" tout au long de la visite (36\% des dyades observés) ;

Conduite 1.2 Les parents qui agissent à la place de l'enfant - ce type d'interaction correspond d'avantage à la catégorie des parents pour lesquels visiter l'exposition consiste à "tout faire" ou "tout faire faire aux enfants" (24\% des dyades) ;

Conduite 1.3 Les parents qui commandent des actions détaillées - Ce type d'attitude déclenche la réalisation d'actions isolées de la part de l'enfant, sans permettre, ni la mise en relation entre l'action et les effets de cette action, ni le questionnement de l'enfant (15\% des dyades) ;

2. Style interactif suggestif :

Conduite 2.1 les parents qui posent des questions à l'enfant pour l'aider et l'accompagner dans la découverte qui l'incitent à faire des liens, à produire des explications ; les parents qui rendent l'enfant actif, reformulent ses questions, précisent le niveau de réussite, demandent l'avis de l'enfant (10\% des dyades) ;

3. Style interactif autonomisant :

Conduite 3.1 Les parents qui laissent l'enfant découvrir seul, qui le soutiennent dans ses essais successifs favorisant ainsi l'apprentissage par essai-erreur ; les parents qui observent tout simplement l'enfant occupé à une tâche, leur présence à côté de lui étant une évaluation implicite, dont le caractère positif émane de l'autorisation accordée à l'enfant de pouvoir poursuivre son activité. (10\% des dyades) ;

4. Style de fonctionnement disjoint

Conduite 4.1 Ce fonctionnement est caractéristique d'une approche individualiste, l'adulte et l'enfant ont chacun leur propre mode d'intervention en le conservant (5\% des dyades).

Encadré 2 : les styles interactionnels et leur prédominance lors de la visite de l'exposition $3 / 5$ ans

Parmi le cinq séquences initiales, trois faisaient référence au style directif, étant donné l'important pourcentage des conduites relatives à ce style $(75 \%)$, une quatrième séquence concernait le style suggestif $(10 \%)$ et une dernière le style autonomisant (10\%). Le style disjoint, peu présent (5\%), n’a pas été proposé.

Les cinq séquences ont été évaluées dans le but d'anticiper leur impact sur les parents et de sélectionner les séquences les plus suggestives, incitant au débat. L'évaluation des séquences c'est faite au moyen d'un questionnaire soumis à trois catégories de public : des étudiants en psychologie (sensibilisés aux interactions adultes-enfants), des parents et des médiateurs scientifiques pour enfants (cf. Tableau 1).

\begin{tabular}{|c|c|c|}
\hline Étudiants en psychologie & Parents d'enfants de 3 à 5 ans & $\begin{array}{c}\text { Médiateurs scientifiques de la Cité } \\
\text { des Enfants }\end{array}$ \\
\hline 52 & 30 & 32 \\
\hline \multicolumn{2}{|c|}{ Total : 114 personnes interviewées } \\
\hline
\end{tabular}

Tableau 1 : Les catégories de public participant à l'évaluation des cinq scénarios

Les items du questionnaire étaient relatifs à la perception des conduites du parent (les répondants étaient invités à se prononcer sur la fréquence de différentes conduites du parent, 
pour chacune des séquences visionnées) et au jugement des conduites du parent en termes d'importance pour l'enfant.

Initialement, avant l'évaluation, nous avions prévu de présenter plus de deux scénarios aux participants pour ne pas leur laisser penser qu'un modèle pouvait être meilleur que l'autre, autrement dit qu'un des deux modèles était à suivre ; les différentes conduites présentées dans les scénarios pouvant être toutes pertinente selon différents facteurs comme leur objectif de visite, la conduite ou l'âge de leur enfant. Finalement seules deux séquences ont été unanimement identifiées par l'ensemble des évaluateurs quant aux conduites qu'elles étaient sensées représenter. Ainsi, lors du débat, l'animateur a portée une attention particulière aux interventions des parents concernant la différenciation des deux scénarios afin qu'ils ne leur attribuent pas une valeur normative (à faire/à ne pas faire). Les extraits de débat qui suivent semblent conforter cette attente, les deux scénarios étant considérés par les parents comme adaptés selon l'importance accordée à l'un ou l'autre des facteurs précédemment cités (cf. Encadré 3 et Encadré 4).

(14) - (...) je pense que ça dépend aussi un petit peu de l'âge, (...) avec Baptiste qui a 4 ans je fais plutôt comme dans le premier film et avec Marie qui a 5 ans, c'est pas du tout la même chose, je pouvais tout à fait lui expliquer comme dans le deuxième...

(17) - peut-être que le sens de passage aussi fait que les enfants sont préparés différemment... il y avait des ateliers préalables qui expliquaient, enfin... je lui avais fait remarquer que lorsqu'on fait couler de l'eau, ça fait tourner donc...

(22) - On peut faire les deux, parce que quand on arrive, on découvre nous-mêmes aussi, on essaie de s'informer, on essaie de faire nous même d'abord, et après on revient pour voir que l'enfant a compris et... je pense que c'est... les deux peuvent exister l'une après l'autre

Encadré 3 : Analyse faite par les parents concernant les conduites vues dans le film

\subsection{Organisation du débat}

$\mathrm{Au}$ cours du débat, les parents sont amenés à adopter une position descriptive et critique par rapport aux situations interactives présentées dans le film. Le débat est organisé selon trois axes :

- la différenciation des types d'interventions - après avoir visionné le film, les parents différencient-ils les interventions et si oui, de quelle manière ?

- l'identification des parents par rapport aux différentes interventions - comment s'identifient-ils par rapport au parent du film ? où se situent-ils ?

- l'évaluation - les participants pensent-ils qu'il existe de meilleures manières d'interagir avec l'enfant que d'autres ? Des extraits de débat sont indiqués dans l'Encadré 4. 
La différenciation des types d'interventions : «Un premier avis, réaction par rapport aux films visionnés? (...) Selon vous, les deux interventions se ressemblent?»

(8) - Parent 5 : La participation de l'enfant n'est pas la même de toute façon. Dans l'un il s'embête carrément, et puis il s'ennuie alors que dans l'autre il est plus... il écoute et puis il reproduit, et puis il essaie d'appliquer un peu...

(9) - Parent $3:(\ldots)$, par rapport au comportement de la mère, le premier était plus réaliste, parce que c'est ce qui se passe $($ rires $) ..$.

(10) - Parent 7 : On lui fait plutôt découvrir dans le deuxième, alors qu'on lui explique, ... on lui explique dans le premier

L'identification des parents par rapport aux différentes interventions : «Par rapport aux films visionnés, pouvez-vous décrire comment êtes-vous intervenu auprès de votre enfant dans cette exposition ? Si vous êtes intervenu d'une autre manière, voulez vous la décrire ?»

(54) - Parent 3 : Je me suis mis à côté de lui et j'ai fais la même chose que lui, ça c'est clair. (...) j’ai trouvé que c'était pas si facile que ça d'expliquer ce qu'il devait faire autrement qu'en lui disant ... “ prends ce bouton et tourne à droite " par exemple... (...) je l'ai fait une fois en lui indiquant les touches et après j'ai essayé de lui refaire faire en lui laissant l'autonomie (...)

(55) - Parent 5 : Moi personnellement c'est vrai que j'ai peut-être un peu tendance à être un peu dirigiste au départ (...) de le récupérer pour ne pas qu'il soit en situation d'échec et au moins lui expliquer le phénomène, si je peux oui...

L'évaluation : « Pensez-vous qu'il existe de meilleures manières d'interagir avec l'enfant que d'autres ?»

(116) - Parent 5 : Faut qu'on s'adapte, si on continue d'expliquer,... l'enfant qu'il en a rien à faire... et puis qu'il joue dans son coin...

(117) - Parent 3 : Parfois peut-être on a tendance à lui en donner trop...

(118) - Parent 5 : Oui, je suis d'accord avec vous... ou lui imposer peut-être...

(119) - Parent $6:(\ldots)$ c'est après avoir compris que je pense avoir été, entre guillemets, meilleure dans la transmission des connaissances.

Encadré 4 : Extraits de débats selon les trois axes de questionnement

Pour aider les participants à identifier les interventions les plus efficaces, les discussions sont orientées par la prise en compte des conduites de l'enfant filmé (ses actions, ses verbalisations, le temps passé sur le dispositif). L'enjeu est de les amener à analyser finement les conduites de l'enfant et à prendre conscience du lien entre la manière dont le parent du film intervient et la conduite de l'enfant. L'animateur du débat s'interdit toute évaluation des propos des participants et toute proposition à caractère normatif. Il se tient à l'écart du débat, son rôle étant de créer un cadre sécurisant de discussion, de mettre à l'aise les participants et de favoriser les échanges entre eux. Dans les extraits rapportés dans l'Encadré 4, on peut ainsi constater que l'animateur du débat n'intervient pas dans les échanges entre participants. Dans le cadre de l'expérimentation du dispositif, l'installation des parents dans une salle attenante à l'espace d'exposition, la présentation du film et la discussion ont duré environ une heure. Le groupe de parents pouvait demander de visionner le film plusieurs fois.

\section{Méthode d'évaluation du dispositif de sensibilisation des parents}

Nous nous attendons à ce que la participation des parents au dispositif déclenche un processus de changement de leurs conduites personnelles, dans le sens d'une meilleure adaptation aux 
besoins de l'enfant dans le contexte muséal. Cette attente est fondée sur l'idée que la discussion centrée sur l'analyse des conduites des acteurs et de leurs relations devrait conduire les parents à des prises de conscience susceptibles de les inciter à être davantage attentifs à la manière dont ils interviennent auprès de leurs enfants. L'évaluation a pour but d'examiner l'évolution des conduites des parents. Pour ce faire, nous avons comparé les interactions parent-enfant avant et après la participation au dispositif de sensibilisation.

\subsection{Comparaison des interactions parent-enfant avant et après l'implication des parents dans le dispositif}

Nous avons choisi un dispositif de référence qui a servi à déterminer le style interactif de chaque dyade : «Orienter l'eau » (décrit brièvement dans la note 1). Il s'agit d'un dispositif qui attire naturellement tous les visiteurs du fait de son emplacement dans l'exposition (c'est un lieu de passage), de son ampleur et de la présence de l'eau en mouvement.

A l'issue de la phase de sensibilisation, chacune des dyades a été filmée dans trois dispositifs :

- le même dispositif qu'en première visite, dispositif qui a été présenté également dans le film (le dispositif muséologique « Orienter l'eau »);

- un deuxième dispositif découvert pour la première fois par la dyade «Les engrenages »...) ;

- un troisième dispositif déjà exploré en première visite par la dyade mais non présent dans le film (« La machine à boules », « Les barrages »);

Compte tenu de la liberté laissée au visiteur ces deux derniers dispositifs peuvent varier d'une dyade à l'autre. La comparaison portant sur le même dispositif, par ailleurs présent dans le film, permet d'apprécier les effets d'imitation éventuelle: les parents ont-ils imité les conduites apparaissant dans le film jugées comme adaptées à l'occasion du débat ? La comparaison concernant le dispositif exploré pour la première fois (une situation similaire à la première visite), permet d'apprécier si la découverte d'un nouveau dispositif muséologique influence le style interactif du parent : dans une situation de première découverte, les parents reproduisent-ils la même conduite qu'avant la séance de sensibilisation, ou bien intègrent-ils les réflexions menées lors de la séance de sensibilisation ? Et enfin, le dispositif déjà exploré et non vu dans le film est considéré comme témoin par rapport à la première comparaison (concernant un dispositif déjà exploré), et permet d'évaluer si les changements des conduites après la séance de sensibilisation se confirment sur les autres dispositifs pour lesquels il n'y a pas de suggestions de conduites, car n'apparaissant pas dans le film. L'ensemble des comparaisons réalisées est synthétisé dans le Tableau 2. 


\begin{tabular}{|c|c|c|c|c|}
\hline $\begin{array}{l}\text { Avant DSP } \\
1 \text { dispositif }\end{array}$ & TIIIISP DIM & 2 & $\begin{array}{l}\text { Après DSP } \\
3 \text { dispositifs }\end{array}$ & \\
\hline $\begin{array}{c}\text { Dispositif } 1 \\
\text { «Orienter l'eau » }\end{array}$ & Orienter reaus & $\begin{array}{c}\text { Dispositif } 1 \\
\text { «Orienter l'eau » } \\
\text { même dispositif }\end{array}$ & $\begin{array}{c}\text { Dispositif } 2 \\
\text { fait pour la 1ère fois }\end{array}$ & $\begin{array}{c}\text { Dispositif } 3 \\
\text { déjà exploré et non } \\
\text { vu dans le film } \\
\text { Témoin du } \\
\text { changement de } \\
\text { conduite }\end{array}$ \\
\hline & & & & \\
\hline
\end{tabular}

Tableau 2 : Les comparaisons (DSP - dispositif de sensibilisation parentale)

\subsection{Population et déroulement de l'étude}

Sept dyades ont été impliquées dans l'étude. Les parents (trois hommes et quatre femmes ; deux employés, trois cadres moyens et deux cadres supérieurs) accompagnaient des enfants âgés majoritairement de quatre ans (cinq garçons âgés de 4 ans et deux filles âgés respectivement de $3 \frac{1}{2}$ ans et 5 ans). Les sept dyades (parents et enfants) découvraient pour la première fois l'exposition. Elles ont été sélectionnées sur la base de leur style interactionnel et recrutées sur la base du volontariat. Les parents sollicités se caractérisent par une prédominance d'actes directifs plus ou moins marqués et à ce titre, ils sont représentatifs des parents qui fréquentent l'exposition (Ailincai \& Weil-Barais, 2003). Dans un premier temps, les parents ont été observés à leur insu. Puis, nous les avons informés que nous faisions une étude sur l'exploitation des jeux par le public et que leur participation les engageait à revenir deux fois. S'ils refusaient, les enregistrements étaient détruits. S'ils étaient intéressés, des visites gratuites leur étaient proposées. Les parents sollicités ont majoritairement répondu favorablement.

Au total, les dyades parent-enfant volontaires pour participer à l'étude ont été filmées à deux reprises, à un mois d'intervalle. Lors des deux visites, les dyades ont été filmées sur plusieurs éléments, les parents ignorant ceux qui nous servaient de référence pour nos analyses.

\section{Exploitation des données}

Les échanges adulte-enfant ont été intégralement transcrits en y ajoutant des aspects non verbaux (gestes, manipulations, mimiques). En effet, les composantes non verbales dans la communication adulte-enfant dans des contextes de jeu sont très importantes (par exemples, les gestes d'encouragement et les mimiques de satisfaction chez l'adulte, la réalisation d'actions chez l'enfant).

Quatre types d'analyses, que nous présentons succinctement, ont été conduites; elles concernent les interventions des parents, la structure des échanges parent-enfant, le niveau de 
distanciation parentale et les tâches proposées à l'enfant. Par ces analyses, il s'agit de rendre compte de la médiation construite par le parent au cours de l'interaction dans ses diverses composantes langagières et pratiques (les tâches effectuées par l'enfant, le plus souvent à la demande de l'adulte).

\subsection{Les interventions des parents}

Une grille d'analyse des interventions verbales et non verbales, qui s'inspire de travaux antérieurs (synthèse réalisée par Adams, 1972), a été construite. Les donnés obtenues par Adams mettent en évidence trois aspects de l'interaction : les comportements centrés sur le contenu à transmettre, les comportements centrés sur la réponse de l'enfant, les comportements de prégnance affective. Les interventions ont été codifiées et analysées selon l'analyse interlocutoire -développée depuis quelques années dans le Laboratoire de Communication de l'Université Nancy 2 sous la direction d'A. Trognon (Ghiglione \& Trognon, 1993)-, et vise à rendre compte du déroulement de la conversation. Ainsi les actes directifs peuvent être apparentés, ou bien au style directif s'ils sont imposés à l'enfant, ou bien au style suggestif si ce dernier en fait la demande.

Le relevé des interventions verbales et non verbales associées aux différents styles d'interaction a permis de cerner le sens de l'évolution des parents en termes de style.

Chaque interaction est caractérisée par un pattern d'interventions (à chaque type d'interventions est associée une fréquence).

\subsection{La structure des échanges}

L'analyse de la structure des échanges s'inspire des travaux relatifs à l'analyse conversationnelle. Les échanges ont été identifiés et caractérisés selon les critères définis par Kerbrat-Orecchioni (1998) : échanges restreints, échanges étendus, échanges tronqués. La proportion d'échanges étendus (caractérisés par plus de trois tours de parole) est un bon indicateur de l'attention conjointe des interlocuteurs et c'est à ce titre que nous l'avons utilisée. De même les échanges tronqués qui se caractérisent par une absence de réaction à une intervention du partenaire et les monologues des parents, indiquent souvent -dans le contexte donné- des difficultés dans la relation éducative.

\subsection{Les tâches}

Dans l'après-coup, en examinant la suite des interventions des parents et des enfants, il est le plus souvent possible d'identifier les objets de leur échange. Nous les avons exprimés en termes de tâches c'est-à-dire en termes d'activités coordonnées par un but explicite ou 
implicite (dénommer, identifier, actionner, comparer, expliquer, etc...) en nous référant à un travail antérieur réalisé par (Ailincai \& Weil-Barais, 2003). Les descriptifs des éléments muséologiques (indiquant les activités possibles que les enfants sont susceptibles de mener) nous ont servi à dresser une première liste de tâches, laquelle a été complétée par l'analyse des interactions. La diversité des tâches dans lesquelles les enfants sont impliqués informe de la richesse des explorations faites.

\subsection{La distanciation parentale}

La distanciation parentale est un modèle emprunté à Sigel (1970, 1982, 1993), repris par de nombreux auteurs, lequel a montré un lien entre le développement intellectuel des enfants et les modes d'intervention des parents. La « distance » se manifeste par tous les comportements ou les événements qui séparent l'enfant de l'environnement immédiat et l'aide à en construire une représentation abstraite. Pour C. Bourdais (2003), reprenant à son compte le modèle de Sigel, les « actes distanciants peuvent être considérés comme des moyens d'étayer la fonction symbolique en ce qu'ils incitent l'enfant à utiliser un mode de représentation plus abstrait tout en tenant compte du niveau actuel de développement; ils assurent davantage qu'un rôle de soutien car ces actes suscitent un mode de représentation que l'enfant n'utilise pas spontanément ». De façon à pouvoir repérer les différents niveaux de distanciation sollicités par les parents, nous avons conçu une grille d'analyse qui prend en compte le contenu propositionnel des actes de langage constituant les interventions des parents. Trois niveaux ont été définis : le niveau «bas » concerne les dénominations, les focalisations de l'attention de l'enfant et les actions non associées à des explications; le niveau «moyen» concerne surtout les comparaisons et les associations d'actions sous-tendues par un même questionnement; le niveau «élevé » concerne les interventions qui activent des processus cognitifs de haut niveau (inférence, évaluation, par exemples). L'intérêt de ce type d'analyse est de disposer d'un moyen d'évaluer les niveaux de traitements cognitifs sollicités par le parent auprès de l'enfant.

\section{Résultats de l'évaluation}

Nous avons comparé les interactions parent-enfant avant et après la participation des parents au dispositif de sensibilisation. Sur la plupart des critères d'analyse considérés, nous observons des changements importants que nous résumons brièvement :

- les actes directifs qui étaient prépondérants chez tous les parents, à des fréquences variables, diminuent quel que soit le dispositif exploré (celui présent dans le film ayant servi de support 
des discussions, un dispositif déjà vu ou un dispositif nouveau) au profit d'actes favorisant l'exploration et l'autonomie de l'enfant. Les fréquences des actes directifs sont récapitulées dans le Tableau 3.

\begin{tabular}{|c|l|c|c|c|}
\cline { 3 - 5 } \multicolumn{2}{c|}{} & $\begin{array}{c}\text { Fréquence } \\
\text { moyenne }\end{array}$ & $\begin{array}{c}\text { Valeur } \\
\text { maximale }\end{array}$ & $\begin{array}{c}\text { Valeur } \\
\text { minimale }\end{array}$ \\
\hline \multirow{2}{*}{ Avant DSP } & « Orienter l'eau » & .71 & .77 & .64 \\
\hline \multirow{3}{*}{ Après DSP } & « Orienter l'eau » & .25 & .44 & .11 \\
\cline { 2 - 5 } & Dispositif témoin & .25 & .33 & .11 \\
\cline { 2 - 5 } & Dispositif nouveau & .22 & .39 & .7 \\
\hline
\end{tabular}

Tableau 3 : Fréquence des actes directifs avant et après la participation au dispositif de sensibilisation parentale (DSP)

- pour l'ensemble des dyades, les échanges tronqués diminuent considérablement ou disparaissent, ainsi que les monologues, en faveur des échanges étendus qui occupent une place prépondérante dans l'interaction, ce qui traduit une amélioration de la communication parent-enfant (cf. Tableau 4).

\begin{tabular}{|c|c|c|c|c|}
\hline & & $\begin{array}{c}\text { Fréquence } \\
\text { moyenne }\end{array}$ & $\begin{array}{c}\text { Valeur } \\
\text { maximale }\end{array}$ & $\begin{array}{c}\text { Valeur } \\
\text { minimale }\end{array}$ \\
\hline \multirow[t]{3}{*}{ Avant DSP } & \multirow{3}{*}{$\begin{array}{l}\text { Echange étendu } \\
\text { Echange Tronqué } \\
\text { Monologue }\end{array}$} & .13 & .36 & .00 \\
\hline & & .14 & .40 & .05 \\
\hline & & .26 & .51 & .06 \\
\hline \multirow[t]{3}{*}{ Après DSP } & \multirow{3}{*}{$\begin{array}{l}\text { Echange étendu } \\
\text { Echange Tronqué } \\
\text { Monologue }\end{array}$} & .65 & .90 & .36 \\
\hline & & .02 & .08 & .00 \\
\hline & & .00 & .00 & .00 \\
\hline
\end{tabular}

Tableau 4 : Fréquence des échanges (étendus, tronqués) et monologues avant et après la participation le (DSP)

- la proportion d'actes distanciants de niveau élevé augmente considérablement d'une visite à l'autre (cf. Tableau 5) ce qui est associé à une baisse des actes distanciants de niveau moyen ou bas lequel disparaît quasiment chez certains parents ; ceci est la traduction d'une meilleure qualité des interventions des parents au plan de la conceptualisation ;

\begin{tabular}{|c|c|c|c|}
\hline Actes niveau élevé & $\begin{array}{c}\text { Fréquence } \\
\text { moyenne }\end{array}$ & Valeur maximale & Valeur minimale \\
\hline Avant DSP & .33 & .40 & .28 \\
\hline Après DSP & .57 & .71 & .45 \\
\hline
\end{tabular}

Tableau 5 : Fréquence des actes distanciants de niveau élevé avant et après la participation au dispositif de sensibilisation parentale (DSP

- concernant les tâches proposées aux enfants, nous n'observons que de légères modifications d'une visite à l'autre : ou bien les parents proposent des tâches nouvelles par rapport à la première visite, ou bien ils abordent les même tâches, mais différemment 
du point de vue du mode de tutelle déployé. Ainsi, les demandes d'actions et d'explications concernant le fonctionnement de l'élément muséologique, ne sont pas les mêmes: lors de la visite avant DSP, les tâches sont réalisées par les parents (manipulations et apports d'explications, descriptions); après DSP, les tâches sont demandées à l'enfant et certains parents accordent une place importante à l'autonomie de l'enfant; les demandes du type «dis-moi », avant DSP, diminuent lors de la deuxième visite, en faveur de demandes de type « montre-moi » (cf. Encadré 5).

Exemple avant le DSP :

Parent : Alors Baptiste, peux-tu m'expliquer... Ce côté-là, explique-moi ce côté-là ! (la mère cherche son regard) (Dyade 7, 25.1)

Parent : Pour que ce moulin vert tourne, dis-moi comment on fait ? (en le retournant vers elle) (Dyade 7, 25.2)

Exemple après le DSP :

Parent : Comment on pourrait en faire tourner deux au bout? (Dyade 7, 27.18)

Parent : J'aimerais bien que tu arrives à en faire tourner deux... le rouge et le bleu là bas ! (Dyade 7, 27.21)

Encadré 5 : Exemples de questions liées à la compréhension du dispositif par l'enfant lors de deux visites

Ainsi, il s'avère que l'impact du dispositif de sensibilisation porte essentiellement sur les modes de tutelle déployés par les parents (appréhendées respectivement à partir de l'analyse des interventions, de la distanciation et de la structure des échanges) et moins sur les tâches proposées à l'enfant. Ceci est sans doute imputable aux orientations données à la discussion entre parents, centrée davantage sur les relations entre interventions du parent et conduite de l'enfant que sur les activités cognitives de ce dernier.

\section{Conclusion et perspectives}

L'évaluation ayant porté sur un échantillon restreint, on se gardera bien d'une conclusion généralisante. Concernant les dyades impliquées dans la recherche, lesquelles n'ont pas manqué de remarquer le caractère exceptionnel de l'expérience à laquelle on leur a demandé de participer, les analyses montrent une évolution marquée et très individualisée entre leur première visite et leur seconde visite au musée. Cette évolution s'observe sur le dispositif de référence qui a servi à la fois à situer les parents par rapport à leur mode d'intervention privilégié et à montrer par le biais d'un film les liens entre les interventions des parents et les conduites des enfants. Ces modifications s'observant également pour un dispositif nouveau, on peut penser qu'il ne s'agit pas d'un effet de mimétisme et de compliance, le parent reproduisant des conduites valorisées lors de la discussion. Tous les critères d'analyse considérés confirment une transformation des interactions parent-enfant. Ainsi peut-on 
légitimement penser que ceci est imputable à la participation au dispositif de sensibilisation, les effets de nouveauté ayant sans doute contribué à amplifier l'effet « dispositif ».

Les changements observés dans le sens d'un meilleur ajustement à l'enfant pourraient s'expliquer par différents facteurs. D'une part, l'absence de normativité lors de la discussion a sans doute contribué à ce que les parents s'engagent dans un processus de changement, sans en être nécessairement conscients, ce qui n'a pas suscité de leur part les résistances parfois rapportées dans d'autres contextes d'éducation parentale. Par ailleurs, la présence d'un film impliquant des acteurs jouant des cas-types d'interaction a permis aux parents de faire le lien entre leurs conduites et celles des enfants; il a facilité les identifications et les prises de distance comme en attestent les analyses des échanges entre parents. Le débat permet d'identifier les modes d'intervention les plus favorables pour l'enfant, sans que les participants ne soient interpellés ou remis en cause.

Le succès du dispositif présenté ici tient sans doute aussi en grande partie aux caractéristiques du public impliqué dans l'étude : des parents soucieux du développement de leur enfant et conscients de leur rôle, non dépourvus par ailleurs de capacités d'analyse comme en attestent les analyses des échanges entre parents au cours de la discussion. Si l'on n'observe pas de différences entre les hommes et les femmes impliquées dans l'étude, l'implication dans le débat n'est cependant pas la même selon les catégories sociales. L'incidence des dimensions sociologiques mériterait donc d'être approfondie.

A l'issue des analyses, les deux éléments-clés du dispositif de sensibilisation des parents nous semblent être confortés : le film, d'une part, la discussion, d'autre part. Le film restitue d'une manière fidèle les conduites des parents observées dans l'exposition; ils montrent des exemples d'interaction parent-enfant qui transmettent beaucoup plus qu'un discours. L'importance de ce film se situe au niveau du débat puisqu'il sert en quelque sorte à guider les parents dans leur analyse : il est support de discussion, moyen de prise de conscience et de distanciation. Nos observations montrent que le film favorise l'expression des émotions et des sentiments, l'identification des situations favorables à l'apprentissage des enfants en fonction des pratiques éducatives des parents ; il permet enfin un apprentissage par imitation. Grâce au film et à la discussion qu'il suscite, les situations éducatives sont interprétées à partir de l'analyse des échanges entre l'adulte et l'enfant à propos de l'activité de celui-ci.

Les discussions entre parents à propos des différents thèmes abordés (l'identification de leurs conduites par rapport aux conduites vues dans le film, l'intérêt de leurs interventions pour 
l'enfant, la compréhension du dispositif par l'enfant, les situations d'apprentissage...) ont influencé différemment leur mode d'intervention après la séance de sensibilisation. Certains parents ont été plutôt sensibles aux types de conduite mobilisés par l'accompagnateur dans l'interaction avec l'enfant, cela se traduisant par une tendance à laisser davantage d'autonomie à leur enfant. D'autres ont été sensibles à la compréhension du dispositif par l'enfant, cela se traduisant par le nombre élevé de tâches proposées à leur enfant lors de la deuxième visite. Enfin, certains parents ont relevé l'importance de l'intervention de l'adulte pour susciter l'intérêt de l'enfant, ce qui s'est traduit en seconde visite par la production de feed-backs réguliers et un ajustement aux conduites de leur enfant. Ainsi les évolutions des parents sont-elles très personnalisées, en lien avec ce qu'ils ont relevé au cours du débat. L'existence d'un lien entre les aspects que les parents relèvent et explicitent au cours de la discussion et les aspects de leurs conduites personnelles qui vont se trouver modifiées en seconde visite atteste de l'importance de la prise de conscience comme levier de changement des conduites personnelles.

On relèvera que la prise en compte des comportements à partir de la lecture du film permet de travailler sur un registre à propos duquel des accords intersubjectifs sont possibles, ce qui est plus difficile lorsqu'on est sur le registre des intentions (par exemple, le parent veut faire comprendre, il veut aider...) ou des valeurs (ce qui est bien ou mal de faire ou de ne pas faire). C'est aussi le plan sur lequel des changements sont attendus.

Par rapport au cédérom interactif conçu par Donald Gordon (1998) - qui demande de réagir, de choisir le bon modèle, et propose un commentaire sur les choix - notre dispositif ne prévoit pas de solution toute faite. Les parents font des hypothèses, les justifient par des exemples issus de leur pratique dans l'exposition ou par des témoignages; ils imaginent ensuite d'autres possibilités éducatives favorables au développement de l'enfant et surtout sont impatients de retourner dans l'exposition pour les tester et les ajuster à la conduite de leur enfant.

Les éléments d'évaluation que nous avons réunis nous encouragent à poursuivre la mise au point de notre dispositif afin de pouvoir l'offrir au public. D'une part, il nous semblerait judicieux d'offrir davantage de scénarios, de façon à prendre en compte la diversité du public et l'ensemble des styles identifiés, cela pour appuyer les témoignages émergeant lors du débat. Au plan des interactions éducatives, il ne peut y avoir de normes ; c'est la souplesse adaptative qui prévaut, orientée par des intentions (faire en sorte que l'enfant s'intéresse à quelque chose, qu'il inhibe son impulsivité, qu'il adapte une posture réflexive, par exemple). 
D'autre part, dans la perspective où les débats seraient conduits par des personnes nouvelles, il conviendrait de mieux spécifier le cadre du débat. Nous ne sommes pas en mesure de savoir actuellement si notre description du cadre est suffisante pour qu'un animateur étranger à l'étude puisse le mettre en place.

Malgré le caractère encore inachevé de la conception du dispositif de sensibilisation parentale présenté dans ce chapitre, il nous semble offrir des perspectives de développement intéressantes qui permettraient de positionner l'éducation parentale dans un espace public dont la mission est d'éduquer tous les citoyens et pas seulement les populations à risque, cibles jusqu'alors privilégiées des programmes d'éducation parentale.

\section{Bibliographie}

Ailincai, R. \& Weil-Barais, A. (2003, avril). Evolution des styles interactifs propres aux parents accompagnant les enfants lors d'une visite sur l'exposition trois/cinq ans. IXème Congrès international de l'AIFREF : La bientraitance dans les différentes cultures, Leuven, Belgique (Actes en ligne sur le site http://w3.umh.ac.be/ pourtois/actes/francais/ailincai.htm)

Ailincai, R., Caillot, M., Weil-Barais, A. (2005, octobre). De l'étude des interactions parents/enfants dans un contexte muséal scientifique à une proposition d'intervention innovante auprès des parents. Les quatrièmes rencontres de l'ARDIST, INRP, Lyon, 2005, pp. 5 - 12

Adams, R. S. (1972). Observational Studies of Teacher Role. Revue Internationale de Pédagogie, 18 (4), 440-459.

Bourdais, C. (2003). Distanciation maternelle et catégorisation dans la deuxième année de la vie. Bulletin de Psychologie, Interaction, acquisition de connaissances et développement, 56(4), 587-597.

Bruner, J.S. (1983). Le développement de l'enfant. Savoir faire, savoir dire. Paris: Presses Universitaires de France.

Cauzinille-Marmèche, E. et Samuels-Pitzini, A. (1996). Interactivité et interactions humaines : quelles interventions à quel moment ? Paris : Cité des sciences et de l'industrie.

Chaumier, S., Casanova, L., \& Habib, M-C. (1995). Les accompagnateurs de la Cité des Enfants. Rapport d'étude soumis au Département Évaluation et prospective, Cité des Sciences et de l'Industrie. Paris.

Ghiglione, R., \& Trognon, A. (1993). Où va la pragmatique ? De la pragmatique à la psychologie sociale. Grenoble : Presses Universitaires de Grenoble.

Gordon, D.A. (1998). Parenting Wisely. Athens (Ohio) : Family Works Inc.

Guichard, J. (1993). La prise en compte du visiteur comme outil de la conception muséologique : un exemple concret, la «Cité des enfants. Public et musées, 3, 111-135.

Guichard, J. (1998). Adapter la muséologie aux enfants. In B., Schiele, \& E., Koster, (Eds.), La révolution de la muséologie des sciences: Vers les musées du XXIe siècle ?. (pp. 207-248). Lyon : Presses Universitaires de Lyon. 
Kerbrat-Orecchioni, C. (1998). Les interactions verbales, tome1. Paris : Armand Colin.

Pourtois, J.-P. (1984), Éduquer les parents ou comment stimuler la compétence en éducation. Bruxelles : Labor.

Pourtois, J. P., Desmet, H. et Nimal, P. (2000). L'éducation familiale à l'épreuve de la nouvelle civilisation. In J-P., Pourtois et H., Desmet, (Eds.), Le parent éducateur (pp. 13-29). Paris : Presses Universitaires de France.

Pourtois, J.-P., \& Desmet, H. (2004). L'éducation implicite. Paris, Presses Universitaires de France.

Sigel, I. E. (1970). The distancing hypothesis : A causal hypothesis for the acquisition of representational thought. In M.R., Jones, (Ed.), Miami symposium on the prediction behavior, 1968 : effects of early experience (pp.99-118). FL : University of Miami Press.

Sigel, I. E. (1982). The relationship between parental distancing strategies and the child's cognitive behavior. In L.M., Laosa \& I.E., Sigel, (Eds.), Families as learning environments for children (pp.47-86). NY and London : Plenum Press.

Sigel, I. E. (1993). The centrality of a distancing model for the development of representational competence. In R. R., Cocking \& K. A., Renninger, (Eds.), The development of meaning of psychological distance (pp. 141-158). Hillsdale, NJ : LEA.

Terrisse, B. (1997), La taxonomie d'objectifs et de soutien parental. St-Sauveur, Québec : Les Éditions du Ponant.

Weil-Barais, A., \& Piani, J. (1998). Comment les parents accompagnent-ils leurs enfants dans la découverte des sciences et des techniques dans un musée ? In A. Dumas Carré \& A. WeilBarais (Eds.), Tutelle et médiation dans l'éducation scientifique. (pp. 251-267) Berne: Peter Lang

Weil-Barais, A., Danis, A., \& Schubauer Leoni, M. L. (2003). Interaction, acquisition de connaissances et développement. Bulletin de Psychologie, 56 (4). 